\title{
LA TRANSITION LATINO-ROMANE : PROBLÈMES DE LA RECONSTRUCTION
}

\begin{abstract}
Summary: Proto-Romance linguistic transformations are partially hidden by the archaic style that characterizes Late Latin documents. However, these texts (e. g. chronicles) permit insights into the changes undergone by the oral language, because authors and scribes can reproduce unconsciously their own speech habits, already different from Classical standard. In our presentation, this curious duality is shown by the example of noun declension, which is undermined, but not yet completely eliminated, in 7th century Latin. A comparison is made between the so-called Fredegarius, a Merovingian chronicle, and an early French poem, the Eulalia Sequence, which manifests the last stage of the declension, just before its disappearance. The morphological change has its counterpart in the restructuration of the sentence: the neighbourhood of subject and verb becomes usual in the surface structure, and certain limitations are imposed upon the freedom of word order. Thus, the reconstruction process we propose has two aspects: it is necessary to describe the diastratic variation at different moments of the history of Late Latin, and, on the other hand, the results need to be compared with the Early Romance linguistic systems. In this manner, reconstruction can show the coexistence of tradition and innovation in the language, a necessary condition of its normal functioning.
\end{abstract}

Key words: Late Latin, declension, linguistic variation

L'étude contrastive des langues consiste, d'une manière générale, à décrire le fonctionnement d'un type d'élément ou d'un type de construction dans un système linguistique, tout en cherchant à dégager, dans un autre système, des éléments et des constructions qui se prêtent à une comparaison structurale avec le point de départ. Cette démarche se pratique couramment en linguistique historique quand on dispose de matériaux suffisants pour représenter les étapes chronologiques successives de l'évolution d'une même langue. Tel est notre procédé lorsque nous voulons rendre compte de l'origine des langues romanes; le problème cependant qui surgit ici, c'est que cette « origine », le latin, s'étale devant nous - à première vue au moins - sous une forme relativement indistincte, tant pour la chronologie précise que pour la stratification sociale de la langue. En effet, nous ramenons beau français et bello italien à bellus sans problème, et de même hermoso espagnol et frumos roumain à formosus - 
mais comment savoir à partir de quel moment ces remplaçants de pulcher deviennent représentants fondamentaux du champ sémantique de la 'beauté' et quel a été leur rapport stylistique mutuel à différents moments de l'évolution ? (La même question peut être soulevée naturellement à propos du couple parabolare / fabulare et beaucoup d'autres.)

Pour répondre à de tels doutes, limitons la distance temporelle, prévue pour la comparaison contrastive, à un intervalle qui semble être arbitrairement choisi, mais qui peut tout de même correspondre à une époque de mutation profonde : il s'agit en gros de la période comprise entre le début du $\mathrm{VII}^{\mathrm{e}}$ siècle et l'apparition des premiers textes romans. Bien sûr, nous rencontrons ici une nouvelle difficulté : c'est que - à partir du $\mathrm{VI}^{\mathrm{e}}$ siècle à peu près - la plupart de nos sources montrent une curieuse dualité. En effet, un discours indubitablement latin - écrit souvent dans un latin de bonne tenue est teinté, pour ainsi dire, d'une couche linguistique plus populaire, car il présente des phénomènes que l'on peut tenter d'interpréter comme les marques du changement linguistique ${ }^{1}$. Cette tentation peut naturellement induire en erreur si notre imagination n'est pas contrôlée par deux considérations. D'une part, les phénomènes nouveaux doivent représenter des ensembles plus ou moins cohérents, reliés, si possible, à d'autres ensembles cohérents; d'autre part, on supposera - mais il n'y a rien là qu'une hypothèse - que les changements observés conduisent vers un état de langue que nous pouvons saisir plus pleinement et qui est celui des premiers documents romans. Ces deux conditions fixées, nous proposons une reconstruction ayant deux aspects : d'une part, nous essayons de retrouver un état du système, une structure qui nous est partiellement cachée à cause des habitudes persistantes de la production textuelle; d'autre part, nous supposons des transformations qui conduisent d'une coupe synchronique à une autre - tout en évitant le piège de prendre nos hypothèses pour des réalités garanties.

En inversant les données du problème, on imaginera une rétrospection à partir du roman ancien jusqu'à ce latin tardif pré-carolingien que l'on vient de proposer comme terme de comparaison. Pour commencer, l'on retiendra la problématique bien connue, pourtant bien complexe, de la déclinaison. Soit le petit poème français de la fin du IX ${ }^{\mathrm{e}}$ siècle, consensuellement appelé la « Séquence de Sainte Eulalie » ${ }^{2}$. Je résume les données relatives à la morphosyntaxe nominale et adjectivale du texte. Les féminins opposent une forme du singulier sans désinence à une forme pourvue de $-S$ final; dans le texte, c'est la marque d'un complément d'objet au pluriel (20 Elle colpes non auret), mais selon le témoignage des autres textes, cet $-s$ indique également, pour les féminins, toutes les autres fonctions du pluriel. Quant aux masculins, ils utilisent le morphème $-s$ comme marque du sujet au singulier (21 pagiens) et comme marque du non-sujet au pluriel (5 mals conselliers, 16 empedemenz, compléments

\footnotetext{
${ }^{1}$ D'un autre point de vue, il s'agit naturellement d'une pénétration de l'usage oral des auteurs et des scribes dans les textes. Pour le degré d' « oralité » des textes écrits, cf. les remarques méthodologiques des éditeurs dans Herring, S. C. - VAn REENEn, P. - SchøSLER, L. : Textual Parameters in Older Languages. Amsterdam-Philadelphia 2000, 17-21.

${ }^{2}$ Édition utilisée : HENRY, A. : Chrestomathie de la littérature en ancien français I. Bern 1953, $2-3$.
} 
d'objet; 7 por ... paramenz, 12 a cels dis, 12 soure pagiens, compléments prépositionnels) et restent sans désinence pour les autres fonctions (non-sujet au singulier : 4 diaule, 6 Deo, 10 menestier, 14 nom christiien, etc. compléments d'objet ; 6 en ciel, 7 ne por or ned argent, 19 enl fou, 25 a ciel compléments prépositionnels, auxquels s'ajoutent les emplois déjà sans doute archaïques pour exprimer la possession ou la destination : 3 li Deo inimi, 10 lo Deo menestier, 11 fut presentede Maximiien; sujet au pluriel : 3 inimi). Le genre apparaît comme bipartite, sans neutre ; le féminin des adjectifs et des participes, dans la mesure où il est distinct du masculin, apparaît comme marqué, étant muni d'un /ə/ « instable »: 1 buona, 11 presentede, 18 morte. Le texte de la "Séquence» fournit un échantillon assez fidèle de la situation des éléments nominaux dans le plus ancien français, situation dont le trait fondamental est la possibilité de marquer le sujet morphologiquement. Sans entrer dans les détails, on peut signaler que ce sont pratiquement les masculins qui opposent un sujet en $-s$ à un non-sujet sans $-s$ au singulier et un sujet sans $-s$ à un non-sujet en $-s$ au pluriel, avec une perte progressive de l'opposition. ${ }^{3}$

Un texte narratif pré-carolingien qui se prête bien à notre étude « contrastive diachronique » et qui illustre en même temps les difficultés de la reconstruction est la chronique dite de Frédégaire, provenant du $\mathrm{VII}^{\mathrm{e}}$ siècle ${ }^{4}$; la tradition manuscrite est ancienne, une des copies remontant au VII ${ }^{\mathrm{e}}$ ou au VIII ${ }^{\mathrm{e}}$ siècle, selon l'éditeur ${ }^{5}$. En parlant de la documentation latine tardive, j'ai dit qu'elle pouvait renfermer des textes de « bonne tenue »- or, Frédégaire n'en est pas un, mais sa formulation linguistique et stylistique offre une dualité caractéristique dont nous pouvons tirer profit. Quand on lit Fr IV 90 (p. 166. 17) Flaochadus ibidem Willebado interfecere nitebatur, on constate la présence d'un trait classique hérité qui deviendra caduc, c'est le déponent; mais on constate également les écarts postclassiques auxquels le rédacteur ou le scribe n'a pas pu résister : interfecere en phonétique et Willebado en morphologie ou plutôt en morphosyntaxe. Pour mieux connaître l'état de la déclinaison, nous pouvons nous appuyer précisément sur cette différence entre les deux « couches » du texte : à un héritage livresque et rigide s'opposent des secteurs en mouvement qui «se cherchent "; or, la déclinaison est l'un de ces secteurs. Dans le chapitre que je suis en train de citer, la disparition du neutre est manifestée par Fr IV 90 (p. 166. 22) Flaochadus deinceps uehementem inibat consilium; en ce qui concerne le système des cas, il reste debout, avec certains décalages bien clairs. La modification la plus saillante est l'abolition d'un trait redondant de l'ancien système : les cas ne respectent plus les automatismes prévus par la grammaire classique, ce qui est d'une part le résultat d'une convergence phonético-morphologique (Fr IV 90, p. 166. 28 de suo

${ }^{3}$ Le conditionnement syntaxique de la disparition de l'opposition morphologique « sujet $\sim$ nonsujet » en ancien français a été étudié par SORNICOLA, R. : Syntactic Conditioning of Case Marking Loss : A Long Term Factor between Latin and Romance? In VAN ACKER, M. - VAN DEYCK, R. - VAN UYTFANGHE, M. (eds.) : Latin écrit - roman oral ? Turnhout 2008, 233-251.

${ }^{4}$ Édition utilisée : Chronicarum quae dicuntur Fredegarii Scholastici libri IV. Ed. BR. KRUSCH. Monumenta Germaniae Historica, Scriptores rerum Merouingicarum II. Hannoverae 1888. (Titre abrégé comme « Fr » par la suite.)

${ }^{5}$ Cf. l'Introduction de l'éditeur (n. 4) 9. Il s'agit du Codex Parisinus Lat. nº 10910. 
intereto et ibid. p. 167. 3 de interetum Villebadi devaient être également prononcés sans $-m$ final), mais se vérifie en dehors de la phonétique par des combinaisons diverses (Fr IV 9, p. 126. 16 imperator Persarum cum sexaginta milia Persus [corr. en Persos] baptizatus est, et per duabus ebdomadis a Iohanne et reliquis episcopis Persas ... baptizantur). Au masculin singulier, on aperçoit la naissance d'un «cas oblique » (non-sujet) généralisé lorsque le génitif est remplacé par une forme en - $o$ : Fr IV 90 (p. 166. 19) germanus Flaochado (pour Flaochadi), ibid. (p. 167. 20) filius Berthario (pour Bertharii) ${ }^{6}$; on peut ajouter d'ailleurs l'emploi de l'expression maiorem domus là où l'on attendrait un génitif: Fr IV 42 (p. 141. 13) secum habens factione Warnachariae (= factionem Warnacharii) maiorem domus ; IV 88 (p. 165. 28) Gradus honoris maiorem domi. Les féminins terminés en - $a$ ne font certainement plus la différence, au singulier, entre nominatif, accusatif et ablatif : Fr IV 9 (p. 126. 8) ipsam esse eorum domina quam querebant; IV 9 (p. 126. 10) uitam illorum instar ad canis est ; IV 10 (p. 126. 25) neglegenciam Bosone (= Bosonis) ... exercitus ille trucidatur; une forme à valeur de 'génitif-datif' semble cependant subsister : Fr IV 90 (p. 166. 15) regnum Burgundiae; ibid. (p. 166. 16) pro utelitate patriae. Un nominatif distinct est maintenu pour les noms de la $3^{\mathrm{e}}$ déclinaison : Fr IV 9 (p. 125. 27) uxor Anaulfi imperatores (= imperatoris) Persarum. Au pluriel, les troubles des constructions absolues permettent de constater le recul de l'ablatif. Un cas clair de «nominatif absolu » (subordonné à une proposition principale) peut être illustré par Fr IV 9 (p. 126. 14) Legati imperatore (= imperatori) Persarum nunciantes, statim ille legationem ad Mauricio imperatore misit; voici une construction où l'accusatif et l'ablatif se mêlent : Fr IV 90 (p. 166. 21) Intercurrentes et citiris persunis ${ }^{7}$.

En respectant les conditions d'interprétation que nous nous sommes fixées, nous pouvons constater d'abord que les phénomènes morphosyntaxiques recensés forment système entre eux, sans que ce système des modifications définisse à chaque coup la forme employée. Nous pensons que le flottement n'est pas un simple reflet du clivage qui existe nécessairement entre langue orale et langue écrite : les rédacteurs et les scribes travaillaient d'après des sources dont ils comprenaient le texte globalement, et ils sont intervenus sur les passages qui leur paraissaient obscurs, ainsi que sur les points où leur système grammatical à eux différait du système traditionnel, mais la modification qui s'imposait était relativement simple. Le traitement des formes casuelles, dont on a présenté ici un échantillon, correspond à ce critère : nous sommes témoins d'une situation où un changement radical est en train de se dérouler, et la variation sociolinguistique - la coexistence d'un système ancien et d'un système nouveau - apparaît de manière aiguë. D'autre part, il faut s'interdire ici toute explication téléologique : les différents traits de la déclinaison que nous avons recensés en latin mérovingien ne conduisent pas nécessairement au système qui se dégage de

\footnotetext{
${ }^{6} \mathrm{Ce}$ « datif possessif », fréquent dans la latinité tardive et caractérisant surtout certains territoires de l'Empire, a été présenté par HERMAN, J. : Le datif possessif dans la latinité balkanique (1965). Étude reprise dans HERMAN, J. : Du latin aux langues romanes : Études de linguistique historique. Tübingen 1990, 315-320.

${ }^{7}$ Dans son ouvrage consacré au langage du « Fredegarius », O. Haag cite plusieurs exemples de cette confusion (HAAG, O. : Die Latinität Fredegars. In Romanische Forschungen 10 [1899] 903).
} 
l'analyse du français médiéval, même si, naturellement, la comparaison permet de se faire une idée du rythme des modifications et d'opérer une coupe synchronique grossière, celle du VII ${ }^{\mathrm{e}}$ siècle en Gaule mérovingienne. On constate ainsi que la décomposition de la déclinaison est bien avancée sur certains points, alors que d'autres structures résistent mieux : la possession peut s'exprimer encore de l'ancienne manière, c'est-à-dire par le génitif, mais celui-ci est concurrencé soit par un génitif-datif (formation nouvelle, mais ayant des racines lointaines), soit par un cas oblique généralisé, auxquels on peut ajouter une autre solution novatrice, notamment l'emploi d'une préposition : Fr IV Capitula 76 (p. 121. 76) De natiuitatem Clodouiae et paccionem de regna cum Austrasius. Les solutions nouvelles persisteront en français médiéval, mais en ce qui concerne la reconstruction elle-même, elle se fonde cette fois sur l'analyse du texte latin ; l'étude contrastive aura lieu ensuite. La comparaison de textes latins de Lombardie avec des documents italiens médiévaux se prête d'ailleurs au même jeu. La "Formula di confessione umbra », texte du $\mathrm{XI}^{\mathrm{e}}$ siècle analysé par Arrigo Castellani, présente régulièrement des sujets sans -s : 11 ipsu D(omi)nideu lo sa; 17 ke lu diabolu n(on) te nde poza accusare 'cosicché il diavolo non ti possa accusare $^{8}$. Les documents rassemblés dans le «Codice Diplomatico Longobardo » témoignent nettement d'un recul du nominatif traditionnel : 16 (I, p. 45. 1) ego Fortonato (Lucca, début du VIII ${ }^{\mathrm{e}}$ siècle) ; 27 (I, p. 100. 23) ego Teutpert clirico (= clericus) (Lucca, VIII ${ }^{\mathrm{e}}$ siècle); 71 (I, p. 216. 16) molestatu fuerit (Toscanella, VIII ${ }^{\mathrm{e}}$ siècle).

Le fonctionnement syntaxique des éléments nominaux possède naturellement un aspect lié à l'arrangement global de la phrase et à sa perspective communicative ; nous touchons là à la question difficilement abordable de l'ordre des termes, dont les contraintes et les libertés sont sensiblement différentes à l'oral et à l'écrit. Dans la « Séquence de sainte Eulalie », selon notre impression générale, il y a des tendances, mais pas de rigidité. La continuité textuelle est bien assurée par la prédominance de l'ordre «sujet (pro)nominal + prédicat verbal»: 9-10 Niule cose non la pouret omque pleier | La polle sempre non amast lo Deo menestier ; 15-16 Il li enortet ... | Qued elle fuiet le nom christiien. Un complément placé en tête de phrase (ici, une anaphore résomptive pronominale) semble demander comme suite immédiate le verbe et le sujet (ordre CVS) : 21 A czo no's uoldret concreidre li rex pagiens; même chose pour un attribut initial : 1 Buona pulcella fut Eulalia. Sur le plan de l'ordre des termes, le texte du «Fredegarius» reste, dans une certaine mesure, dans les cadres classiques et ne s'écarte pas beaucoup de l'un des schémas hérités, où les phrases narratives ont tendance à commencer par le sujet (souvent précédé par une détermination temporelle): Fr IV 90 (p. 166. 22) Flaochadus deinceps uehementem inibat consilium de interetum Villebadi. Eo anno Nantildis regina moritur. Le verbe, généralement rhématique, se place en fin de phrase ou, plus rarement, avance vers le début, en cherchant le voisinage du sujet; nous avons alors souvent un rhème complexe du type $\mathrm{VC}$, comme ici, avec inibat + consilium + de interetum Villebadi ou dans

\footnotetext{
${ }^{8}$ Pour ce texte et sa traduction, cf. CASTELLANI, A. : I più antichi testi italiani-edizione e commento. Bologna 1980, 77-102.

${ }^{9}$ Les chartes rédigées en Italie lombarde entre les VII $-\mathrm{VIII}{ }^{\mathrm{e}}$ siècles ont été publiées par SCHIAPARELLI, L. : Codice Diplomatico Longobardo I-II. Roma 1929-1933.
} 
Fr IV 33 (p. 133. 6) Eo anno, mortuo Bettarico, Sisebodus Spaniae successit in regno, uir sapiens ... pietate plenissemus (avec une rallonge postverbale considérable). Virtuellement, la mobilité des termes qui caractérise le latin classique subsiste, et un rhème nominal fortement accentué peut suivre le verbe, en amenant, en début de phrase, un ordre VS : Fr IV 15 (p. 127. 21) apparuit stilla comitis (= cometes). Certaines données provenant de textes assez différents semblent indiquer une tendance à rapprocher sujet et prédicat verbal, soit sous la forme $\mathrm{SV}:$ Egeria $^{10}$ 3. 7 Tunc statim illi sancti dignati sunt singula ostendere; Hydatius ${ }^{11} 83$ Iohannes arripit tyrannidem; soit sous la forme VS : Egeria 3.4 Occurrerunt etiam et alii presbyteri : Hydatius 127 Constantinopolitanae ecclesiae depulso Nestorio praesidet episcopus Flauianus - ce dernier exemple représentant par ailleurs une phrase type, à laquelle le chroniqueur Hydatius a systématiquement recours pour noter l'attribution des dignités ecclésiastiques ${ }^{12}$. Ainsi, du point de vue «contrastif», nous pouvons opposer une souplesse et, pour ainsi dire, une sorte d'expérimentation caractérisant le latin tardif, à une fixité d'un type nouveau qui semble se dessiner en ancien français, à travers des hésitations, avec SVC et CVS comme arrangements dominants.

Du point de vue de la reconstruction des réalités linguistiques anciennes, une conclusion méthodologique se dessine assez nettement. L'espèce de dualité ou - pour utiliser un terme plus général - l'empreinte de la variation diastratique que nous devons envisager dans les textes ${ }^{13}$ nous invite à reconstruire, au-delà de systèmes ou de sous-systèmes synchroniques, une sorte de réalité dynamique, caractérisée par des changements en cours et des tendances en profondeur. L'état de la déclinaison, tel qu'il se présente dans le texte des chroniqueurs gallo-romans, se caractérise avant tout par une recherche, celle d'un cas oblique unifié, opposé au nominatif et assisté par des prépositions, pour les masculins, et celle d'un fonctionnement sans marque casuelle pour les féminins, avec manipulation des prépositions ici aussi ${ }^{14}$. Reprenons ici Fr IV 9 (p. 126. 10) uitam illorum instar ad canis est 'leur vie est pareille à celle des chiens', phrase qui témoigne encore une fois de la disparition d'une opposition fondamentale dans le paradigme de uita et qui introduit en même temps une préposition à la place de la construction classique demandant le génitif avec instar. Si nous avons réussi à saisir les traces d'une telle variation sociolinguistique, nous devons essayer de voir si l'évolution soupçonnée derrière la variation peut être mise en rapport avec d'autres flottements, sans qu'il s'agisse nécessairement d'une relation de cause à effet : des évolutions parallèles peuvent se stimuler mutuellement. En ce qui concerne le problème

\footnotetext{
${ }^{10}$ Itinerarium Egeriae (Peregrinatio Aetheriae). Ed. O. PRINZ. Heidelberg 1960 ${ }^{5}$.

${ }^{11}$ Hydatii Lemici continuatio chronicorum Hieronymianorum. Ed. TH. MommSEN. Monumenta Germaniae Historica, Auctores Antiquissimi XI. Berolini 1894.

${ }^{12}$ Cf. KISs, S. : Les documents latins du Haut Moyen Âge et la naissance du français $I:$ La chronique d'Hydatius [Studia Romanica de Debrecen, Series Linguistica, fasc. X]. Université de Debrecen 2006, 13.

${ }^{13}$ Pour une caractérisation concise et précise de cette variation dans le préroman de la Gaule, cf. $\mathrm{KoCH}, \mathrm{P} .:$ Le latin - une langue pas tout à fait comme les autres ? Le problème de la diglossie en Gaule septentrionale. In VAN ACKER - VAN DEYCK - VAN UYTFANGHE (n. 3) 43-67.

${ }^{14}$ Pour une vue générale de l'emploi des formes casuelles et des constructions prépositionnelles en latin, cf. ADAMS, J. N. : Social Variation and the Latin Language. Cambridge 2013, 257-260.
} 
concret qui nous occupe ici, on peut penser naturellement à une tendance qui se laisse deviner dans l'arrangement général de la phrase : est-ce que le rapprochement du sujet et du verbe correspond à un besoin de clarté accru, d'autant plus plausible que la ruine de la déclinaison devait être compensée par une transformation structurale qui faisait prévoir certains schémas probables dans la communication? Naturellement, une telle transformation syntaxique pouvait se mettre en place indépendamment de l'état de la déclinaison, et dans ce cas, elle pouvait favoriser la simplification morphologique, qui constituait en latin tardif une tendance répandue. On peut en dire autant du rôle du facteur phonologique. La disparition de $-m$ final fait partie d'un ensemble de changements plus vaste qui touchent la structure de la syllabe et la phonologie du mot en latin postclassique ; dans son interaction avec la morphosyntaxe, elle pouvait contribuer à l'élargissement de l'emploi des prépositions ou à la transformation de l'ordre des mots, mais elle pouvait être elle-même accélérée par l'extension de certaines solutions syntaxiques ${ }^{15}$.

En guise de conclusion, on peut dire que dans l'état actuel de la recherche, ce que l'on doit reconstruire, ce ne sont pas d'abord l'inventaire des phonèmes, les formes grammaticales et les vocables, mais les tendances de l'évolution et les rapports qui les relient, ainsi que les « interfaces » phonologie / grammaire et grammaire / lexique. D'autre part, notre reconstruction doit tenir compte de la coexistence de différents sociolectes, et elle doit montrer le nécessaire équilibre entre conservation et innovation, sans lequel le fonctionnement normal de la langue ne serait pas possible.

\author{
Sándor Kiss \\ Département de Français \\ Université de Debrecen \\ Hongrie \\ kiss.sandor@arts.unideb.hu
}

Open Access. This is an open-access article distributed under the terms of the Creative Commons Attribution 4.0 International License (https://creativecommons.org/licenses/by/4.0), which permits unrestricted use, distribution, and reproduction in any medium, provided the original author and source are credited, a link to the CC License is provided, and changes - if any - are indicated. (SID_1)

\footnotetext{
${ }^{15}$ L'hypothèse selon laquelle les changements morphosyntaxiques et les changements phonologiques du latin tardif se sont influencés mutuellement est une hypothèse ancienne. Ainsi, JUNGEMANN, F. H. (La teoría del sustrato y los dialectos hispano-romances y gascones. Madrid 1955, 310-314), en cherchant les origines de ces transformations, refuse de donner la priorité absolue au facteur phonologique. De toutes manières, le système phonologique et le système grammatical ont leurs propres tendances d'évolution, guidées par les besoins d'un équilibre interne qui sert la communication efficace. Pour la chute des consonnes finales de mot et la recherche des syllabes ouvertes en latin postclassique, cf. KISS, S. : Les transformations de la structure syllabique en latin tardif [Studia Romanica de Debrecen, Series Linguistica, fasc. II]. Université de Debrecen 1972, 89.
} 
